Article

\title{
Cysteine Residues in Helicobacter pylori Adhesin HopQ Are Required for CEACAM-HopQ Interaction and Subsequent CagA Translocation
}

\author{
Youssef Hamway ${ }^{1}$, Karin Taxauer ${ }^{1}$, Kristof Moonens ${ }^{2,3}$, Victoria Neumeyer ${ }^{1}$, \\ Wolfgang Fischer ${ }^{4}$, Verena Schmitt ${ }^{5}$, Bernhard B. Singer ${ }^{5}(\mathbb{D})$, Han Remaut ${ }^{2,3}{ }^{\mathbb{D}}$, \\ Markus Gerhard ${ }^{1,6}$ (D) and Raquel Mejías-Luque 1,6,*(D) $^{-1}$ \\ 1 Institute for Medical Microbiology, Immunology and Hygiene, Technical University Munich, 81675 Munich, \\ Germany; youssef.hamway@tum.de (Y.H.); karin.taxauer@tum.de (K.T.); victoria.neumeyer@tum.de (V.N.); \\ markus.gerhard@tum.de (M.G.) \\ 2 Structural and Molecular Microbiology, VIB-VUB Center for Structural Biology, VIB, 1050 Brussels, Belgium; \\ kristof.moonens@gmail.com (K.M.); han.remaut@vub.be (H.R.) \\ 3 Department for Bioengineering Sciences, Structural Biology Brussels, Vrije Universiteit Brussel, \\ 1050 Brussels, Belgium \\ 4 Med. Mikrobiology and KH-Hygiene, Max von Pettenkofer-Institute, Ludwig-Maximilians-Universität, \\ 80336 Munich, Germany; Fischer@mvp.lmu.de \\ 5 Institute of Anatomy, Medical Faculty, University of Duisburg-Essen, 45147 Essen, Germany; \\ BBSINGER@gmx.de (V.S.); verena.schmitt@uk-essen.de (B.B.S.) \\ 6 German Center for Infection Research (DZIF), partner site Munich, 81675 Munich, Germany \\ * Correspondence: raquel.mejias-luque@tum.de
}

Received: 12 February 2020; Accepted: 24 March 2020; Published: 25 March 2020

\begin{abstract}
Attachment to the host gastric mucosa is a key step in Helicobacter pylori infection. Recently, a novel adhesin, HopQ, was shown to bind distinct host CEACAM proteins-an interaction that was found to be essential for the translocation of CagA, a key virulence factor of H. pylori. The HopQ-CEACAM1 co-crystal structure revealed a binding mode dependent on loops in HopQ that are clasped by disulfide bonds. In this study, we investigated the importance of these cysteine residues for CEACAM1 engagement by $H$. pylori. We observed a loss of CEACAM1 binding and CagA translocation upon disruption of the disulfide bond in loop CL1 (connecting C103 to C132 in HopQ). Deletion of the Dsb-like oxidoreductase HP0231 did not affect cell surface expression of HopQ or alter the interaction of H. pylori with target cells. Although HP0231 deletion was previously described to impede CagA translocation, our results indicate that this occurs through a HopQ-independent mechanism. Together, our results open up new avenues to therapeutically target the HopQ-CEACAM1 interaction and reduce the burden of pathogenic H. pylori.
\end{abstract}

Keywords: bacterial adhesion; CagA delivery; CEACAM1; Helicobacter pylori; HopQ; host-pathogen interactions; Dsb-like proteins

\section{Introduction}

Infection with the gastric pathogen Helicobacter pylori is one of the most prevalent infections worldwide. H. pylori is considered a class I carcinogen, as the infection is one of the main risk factors for gastric cancer development [1]. In order to colonize and persist in the stomach, H. pylori needs to bind to gastric epithelial cells. A major mechanism of $H$. pylori adhesion to host cells involves the interaction between the BabA adhesin and Lewis b receptors on the gastric mucin MUC5AC [2]. However, over recent years, other important $H$. pylori adhesins have been identified, for example the outer membrane 
adhesin HopQ that binds to CEACAM receptors expressed on the gastric epithelium [3,4]. The binding of HopQ to CEACAM was found to be essential for H. pylori pathogenesis, as it is required for the type 4 secretion system (T4SS)-mediated translocation of the key virulence factor CagA into gastric epithelial cells $[3,4]$.

Whereas BabA is a lectin that mediates binding to host carbohydrates, the HopQ-CEACAM1 structure highlighted the importance of direct protein-protein contacts for its interaction $[3,5]$. Interestingly, the co-complex structure revealed the presence of two cysteine-clasped loops (CL1 and CL2) that cluster together at the tip of the HopQ adhesin domain to form a platform of contact with the N-terminal domain of CEACAM1 [5,6]. Both loops are anchored by a pair of conserved cysteines. These may affect structural stability of the binding region and thereby influence attachment to CEACAM1 [5]. Other reports highlighted the importance of disulfide bond formation on the functionality of H. pylori virulence factors, such as the T4SS and VacA [7], and the importance of the cysteine-clasped loop in the glycan binding site of the BabA adhesin [8].

In Gram-negative bacteria, the formation of disulfide bonds occurs in the periplasm and is controlled by membrane-bound Dsb proteins [9]. The function of Dsb proteins has been extensively studied in Escherichia coli [9], where DsbA and DsbC play an essential role in disulfide bond formation $[10,11]$. In contrast, $H$. pylori possesses a relatively simple system, since it does not encode classical DsbA/DsbB or DsbC/DsbC oxidoreductases. In fact, only two extra-cytoplasmic Dsb proteins, HP0377 and HP0231, have been identified [12-14]. HP0377 is involved in cytochrome-c maturation and possesses disulfide isomerase activity in vitro [14]. HP0231, a major dimeric oxidoreductase of H. pylori, catalyzes disulfide bond formation in the periplasm [13]. HP0231 is not only important for the maintenance of redox homeostasis in $\mathrm{H}$. pylori, but is also essential for the proper function of virulence factors possessing disulfide bonds. It has been reported that H. pylori lacking HP0231 are unable to translocate CagA into gastric epithelial cells [7]. In addition, VacA-mediated vacuolation is impaired upon loss of HP0231 expression and, more importantly, HP0231 is required for gastric colonization [7].

As HopQ-mediated binding to CEACAM1 involves the cysteine-clasped loops CL1 and CL2, we investigated whether the cysteines in these loops were important for HopQ stability and function. HopQ interaction with CEACAM receptors has been described as being essential for CagA translocation $[3,4]$ and so we investigated whether the oxidoreductase HP0231, that was shown to be critical for CagA translocation, is involved in HopQ disulfide bond formation. Our results indicate that disruption of the disulfide that stabilizes CL1 abolishes HopQ-mediated CEACAM binding. However, we found that HopQ expression and adherence to host CEACAM receptors are independent of HP0231.

\section{Materials and Methods}

\subsection{H. pylori Strains and Generation of Mutant Strains}

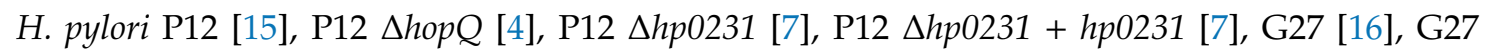
$\Delta h o p Q[3]$ and G27 $\Delta h p 0231$ [7] were used in this study.

To generate H. pylori complemented with wild type HopQ or point mutants, HopQ was cloned into the pWS241 vector using Gibson Assembly (NEB), and point mutants (C103S, C132S, C238S and C362S) of the gene generated using the Quikchange system (Agilent, Santa Clara, CA, USA). The resulting plasmids were transformed into $H$. pylori $\mathrm{G} 27 \Delta$ hopQ. In brief, bacteria grown on WC Dent plates for two days were collected and resuspended in Brain Heart Infusion (BHI) medium, $2 \mu \mathrm{g}$ of plasmid were added to $200 \mu \mathrm{l}$ of this suspension, mixed, replated on WC Dent plates, and allowed to grow for $24 \mathrm{~h}$. Bacteria were then transferred to WC Dent plates containing $50 \mu \mathrm{g} / \mathrm{mL}$ kanamycin to select for bacteria harboring the plasmid. The expression of HopQ was verified by Western blot (Figure 2a). 


\subsection{Cells}

MKN28 [17] vector control, MKN28-CEACAM1-4L, AGS (ATCC, CRL-1739), CHO (ATCC, CCL-61) vector control, and CHO-CEACAM1-4L cells [3] were maintained in an incubator at $37^{\circ} \mathrm{C}$ and $5 \% \mathrm{CO}_{2}$. Cells were grown in DMEM (Gibco, Thermo Fisher Scientific, Waltham, MA, USA) containing $2 \mathrm{mM}$ L-glutamine (Gibco) supplemented with 10\% FBS (Gibco) and 1\% penicillin/streptomycin (Gibco).

\subsection{Infections and Western Blot for CagA Translocation}

Cells were seeded at a density of $7.5 \times 10^{4}$ in a 24-well plate, two days before infection. On the day of the infection, one well was trypsinized and harvested cells were counted.

H. pylori grown on WC Dent plates for two days were collected, resuspended in BHI medium, and counted based on their optical density $\left(\mathrm{OD}_{600}\right)$, with $\mathrm{OD}_{600}=1=2 \times 10^{8}$ bacteria. The number of bacteria required for a multiplicity of infection (MOI) of 50 was determined, added to the eukaryotic cells and incubated for $6 \mathrm{~h}$ at $37^{\circ} \mathrm{C} / 5 \% \mathrm{CO}_{2}$. After infection, the cells were washed $1 \mathrm{x}$ with PBS, lysed with Laemmli buffer + 50mM DTT, and run in an SDS-PAGE on $6 \%$ polyacrylamide gels followed by a Western blot onto nitrocellulose membranes (GE Healthcare, Chicago, IL, USA). The Western blot membrane was blocked with 5\% skim milk (Carl Roth, Karlsruhe, Germany)/PBST probed with mouse anti-pTyr (1:300), rabbit anti-CagA serum (1:3000), and rabbit anti-GAPDH (1:1000) or anti-alpha tubulin (1:1000, loading control) in 5\% Bovine serum albumin (Applichem, Darmstadt, Germany)/PBST. The CagA blot was reprobed with the anti-pTyr antibody to detect phosphorylated CagA.

\subsection{Pull Down Assays}

H. pylori grown on WC Dent plates for two days were collected and their optical density measured. The cultures were adjusted to $\mathrm{OD}_{600}=0.5 \mathrm{in} 1 \mathrm{~mL}$ of media, and $2 \mu \mathrm{g}$ CEACAM1-Fc recombinant protein [18] was added. The mixture was left to shake for $2 \mathrm{~h}$ in a microaerobic incubator, followed by washing $3 \times$ with PBS. The washed sample was lysed in Laemmli buffer + $50 \mathrm{mM}$ DTT, run in an SDS-PAGE followed by a Western blot and the membrane was probed for CEACAM1 (SAB3 antibody, $2.5 \mu \mathrm{g} / \mathrm{mL}$ ) and HopQ (mouse serum, 1:3000) as a control.

\subsection{Binding Assay (Flow Cytometry)}

H. pylori grown on WC Dent plates for two days were collected and their optical density measured. The cultures were adjusted to $\mathrm{OD}_{600}=1 \mathrm{in} 1 \mathrm{~mL}$ of media and stained with $10 \mu \mathrm{M}$ Carboxyfluorescein succinimidyl ester (CFSE, eBioscience, San Diego, CA, USA) for $30 \mathrm{~min}$. The bacteria were washed twice with PBS, counted once more, and added to AGS or pcDNA3.1 empty vectoror CEACAM1-4L-transfected MKN28 or CHO cells at MOI 10. The mixture was incubated in a microaerobic incubator, shaking for $30 \mathrm{~min}$; followed by washing four times with PBS. After washing, the samples were resuspended in 1\% PFA/PBS and acquired on a Cyan ADP or CytoFLEX S flow cytometer (Beckman Coulter, Brea, CA, USA). The eukaryotic cells were gated based on forward and side scatter, and their fluorescence measured in the FITC channel. This quantified the proportion of the gated cells bound by the fluorescent bacteria. $100 \%$ indicates that each eukaryotic cell exhibited fluorescence H. pylori binding.

\subsection{Immunofluorescence}

$1.5 \times 10^{5}$ MKN28-CEACAM1-4L or CHO-CC1 were seeded on coverslips in a 12-well plate and grown for two days. H. pylori grown on WC Dent plates for two days were collected, counted and CFSE-stained as described above. The CFSE-labeled bacteria were then used to infect the cells at MOI 10 for $3 \mathrm{~h}$. After infection, the cells were methanol/acetone-fixed, phalloidin-stained and mounted with DAPI-containing medium (Vector Labs, Burlingame, CA, USA). Pictures were taken using a confocal microscope SP5 (Leica, Wetzlar, Germany). 


\subsection{Thermal Denaturation Assay}

Solutions of $7.5 \mu \mathrm{L}$ of $300 \times$ SYPRO Orange (Invitrogen, Carlsbad, CA, USA), $5 \mu \mathrm{L}$ of $2.5 \mathrm{mg} / \mathrm{mL}$ HopQ type I adhesin domain (HopQ ${ }^{\mathrm{AD}-\mathrm{I}}$, comprising residues $22-425$ of the mature protein, for production details see [5]) and $12.5 \mu \mathrm{L}$ of a dithiothreitol (DTT) dilution series or buffer (20 mM Tris $\mathrm{HCl} \mathrm{pH} \mathrm{8.0,} 150 \mathrm{mM} \mathrm{NaCl}$ and 5\% (v/v) glycerol) were added to the wells of a MicroAmp Optical 96 well Reaction Plate (Applied Biosystems, Foster City, CA, USA). The plates were sealed with Microamp optical adhesive film (Applied Biosystems) and heated in a 7500 Real Time PCR System (Applied Biosystems) from 4 to $95^{\circ} \mathrm{C}$ in increments of $1^{\circ} \mathrm{C}$. Fluorescence changes in the wells of the plate were monitored simultaneously and plotted against temperature. The wavelengths for maximal excitation and emission of the SYPRO orange are 470 and $570 \mathrm{~nm}$, respectively.

\subsection{CEACAM-GFP Binding Assay}

H. pylori grown on WC Dent plates for two days were collected and their optical density measured. The cultures were adjusted to $\mathrm{OD}_{600}=1$ in $1 \mathrm{~mL}$ of media and stained with $5 \mu \mathrm{M}$ Cell Proliferation Dye eFluor ${ }^{\mathrm{TM}} 670$ (eBioscience) for $30 \mathrm{~min}$. The bacteria were washed twice in PBS, and evenly divided into 8 wells of a 96 well plate, to which $3 \mu \mathrm{g}$ of the respective CEACAM-GFP (or GFP control) [19-21] were added. The mixture was incubated in a microaerobic incubator, and shaken for $30 \mathrm{~min}$; followed by washing $4 \mathrm{x}$ with PBS. After washing, the samples were fixed in 1\% PFA/PBS and acquired on a CytoFLEX $S$ flow cytometer.

\subsection{H. pylori Outer Membrane Preparation}

H. pylori strains were grown as described above, then $100 \mathrm{mg}$ of bacteria was collected and resuspended in $300 \mu \mathrm{l}$ of buffer $(20 \mathrm{mM}$ Tris, $500 \mathrm{mM} \mathrm{NaCl}$, pH $8+2 \mathrm{mM}$ EDTA, $100 \mu \mathrm{g} / \mathrm{mL}$ lysozyme, $20 \mu \mathrm{g} / \mathrm{mL}$ DNAase and cOmplete ${ }^{\mathrm{TM}}$, Mini, EDTA-free Protease Inhibitor Cocktail (Roche, Basel, Switzerland)). This was followed by $4 \times$ freeze-thaw cycles using dry ice and a water bath to lyse the bacteria. The lysed bacteria were then centrifuged at $10,000 \times g, 4{ }^{\circ} \mathrm{C}$ for $15 \mathrm{~min}$. The supernatant was collected and centrifuged at $20,000 \times g, 4^{\circ} \mathrm{C}$ for $90 \mathrm{~min}$. The pellet was retained and dissolved in $200 \mu \mathrm{L}$ of the above buffer $+0.5 \% \mathrm{~N}$-lauroylsarcosine and incubated for $30 \mathrm{~min}$ at $\mathrm{RT}$, shaking. This solution was centrifuged once more at $20,000 \times g, 4{ }^{\circ} \mathrm{C}$ for $90 \mathrm{~min}$. The pellet was retained and dissolved in Laemmli buffer $+50 \mathrm{mM}$ DTT, run in an SDS-PAGE followed by a Western blot and the membrane was probed for HopQ as above, with HpaA as a positive control (mouse serum, 1:3000) and gGT as a negative control (mouse serum, 1:3000).

\subsection{List of Antibodies Used}

Mouse anti-pTyr (1:300, PY99, Santa Cruz Biotechnology, Dallas, TX, USA);

Rabbit anti-CagA serum (1:3000);

Rabbit anti-GAPDH (1:1000, loading control, 14C10, Cell Signaling Technology, Danvers, MA, USA);

Rabbit anti-alpha tubulin (1:1000, loading control, polyclonal, Cell Signaling Technology);

SAB3 antibody (anti-CEACAM1/3/5 from B.B. Singer), $2.5 \mu \mathrm{g} / \mathrm{mL}$;

Mouse anti-HopQ serum (1:3000);

Mouse anti-HpaA serum (1:3000);

Mouse anti-gGT serum (1:3000).

\section{Results}

\subsection{Disulfide Bridges in the HopQ Adhesin Domain are Essential for CEACAM1 Binding}

To assess whether disulfide bond formation is important for HopQ stability, we performed a thermal stability (Thermofluor) assay on the HopQ adhesin domain (HopQ ${ }^{\mathrm{AD}}$, comprising residues $22-425$ of the mature protein) in the presence of different concentrations of the reducing reagent 
dithiothreitol (DTT). We observed that even small amounts of DTT (1-2 mM) were able to destabilize HopQ $\mathrm{Q}^{\mathrm{AD}}$, as evidenced by the shift of the Thermofluor peak (Figure 1). Increasing concentrations of DTT even further reduced HopQ ${ }^{\mathrm{AD}}$ thermal stability until around $30^{\circ} \mathrm{C}$, where a plateau was reached in the range of 10-100 $\mathrm{mM}$ DTT.

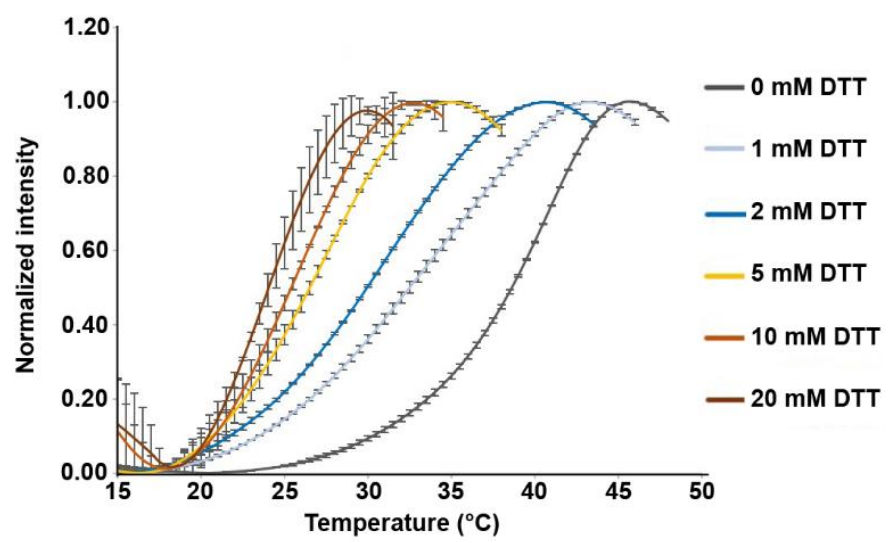

Figure 1. Disulfides are critical for HopQ stability. Thermal shift (Thermofluor) assay measuring the thermal unfolding of HopQ in the presence of a range of DTT concentrations. Error bars show S.D.

Our results indicate that HopQ stability depends on disulfide bond formation. Therefore, loss of disulfides might impact HopQ functionality. HopQ ${ }^{A D}$ possesses three different cysteine-clasped loops CL1 (anchored by residues C103 and C132); CL2 (anchored by residues C238 and C270); and CL3 (anchored by residues C362 and C385), of which CL1 and CL2 were likely to influence HopQ binding activity as deduced from the HopQ-CEACAM1 co-complex structure [5]. To test this hypothesis, we generated cysteine to serine mutant strains C103S, C132S, C238S and C362S (from CL3, as a negative control), and tested binding to CEACAM1 in pull down experiments. We observed that CL1 mutants (C103S and C132S) reduced CEACAM1 binding to the levels of the $\triangle$ hopQ mutant (Figure 2a), while the CL2 and CL3 mutants (C238S and C362S) had no effect on the pull-down of CEACAM1 (Figure 2a). Of note, reduced surface expression of the C132S mutant was detected, which could account for the reduced binding to CEACAM1 that was observed for this strain (Figure 2b); however, this is in keeping with the overall reduced expression of HopQ by this strain (Figure 2a). In contrast, similar levels of HopQ were observed in membrane preparations of the C103S mutant compared to the wild type strain (Figure 2b). This suggests that the observed reduction in binding is based on these mutations altering the direct interaction of HopQ to CEACAM1, rather than being a result of structural changes that prevent outer membrane expression of the protein. Together, these results suggest an important role for the CL1 disulfide bridge in CEACAM1 binding.

We next performed binding assays to $\mathrm{CHO}$ cells stably transfected with CEACAM1-4L (CHO-CEACAM1-4L) [22]. A parental CHO cell line that does not express any CEACAM proteins was used as a negative control. In agreement with the loss of CEACAM1 interaction observed with the pulldown assay (Figure 2a), flow-cytometry-based binding assays showed that the binding of H. pylori cells expressing HopQ mutants C103S or C132S to CHO-CEACAM1 cells was reduced to the levels seen for the $\Delta h o p Q$ mutant (Figure 2c). Similar results were obtained when using MKN28 cells (Figure 2d), thus confirming the importance of the CL1 disulfide on a cellular level. We also confirmed these observations using confocal microscopy (Figure 2e). Together, these results indicate that disulfide bond formation, specifically in CL1, is critical for HopQ stability and function.

HopQ has also been shown to bind CEACAM5 [3]. To determine whether disulfide formation would also affect the binding of $H$. pylori to CEACAM5, we tested binding of the various cysteine mutants to recombinant CEACAM5-GFP. We found that loss of the CL1 disulfide bond similarly affected binding (Figure 2f). 
(a)

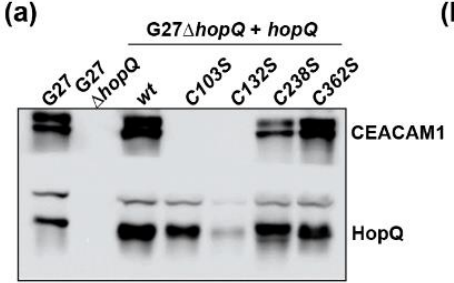

(b)

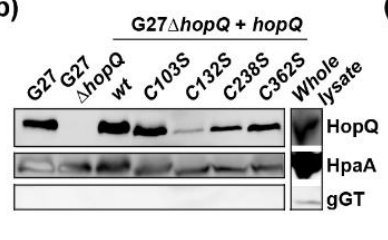

(c)

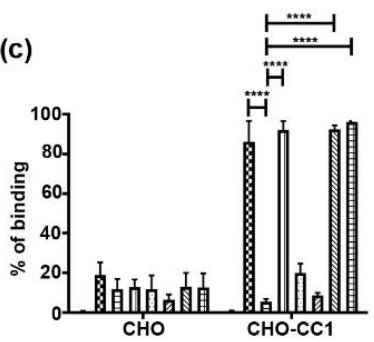

日G27 $\triangle h O p Q$

四27 $\triangle h o p Q+h o p Q$

$\square \mathrm{G} 27 \Delta h \mathrm{OPQ}+\mathrm{hop}^{\mathrm{c} 103 \mathrm{~s}}$

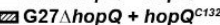

G27 $270 p Q+h o p Q c 23$

四27 $h \circ Q p Q+h o p Q^{c 362 s}$
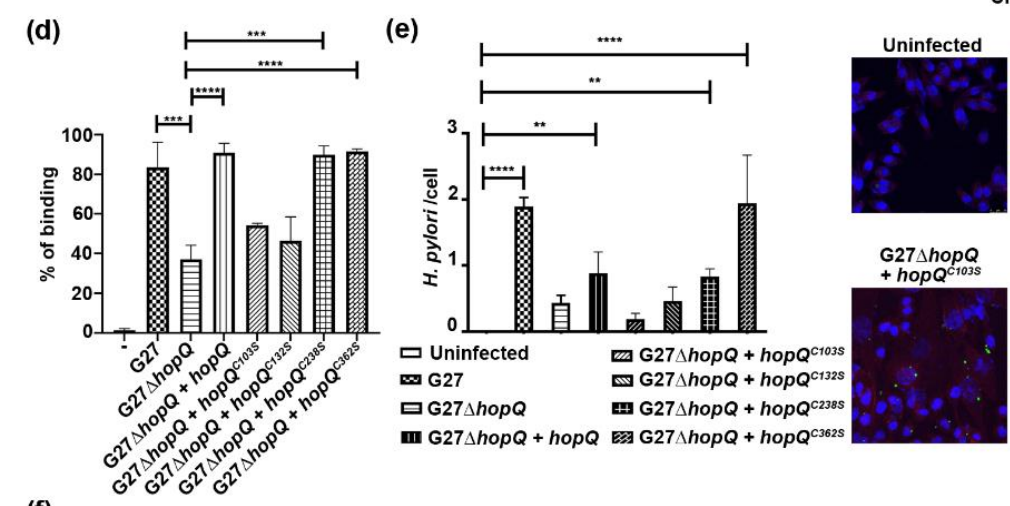

G27

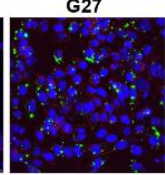

G27 $\triangle h O p Q$
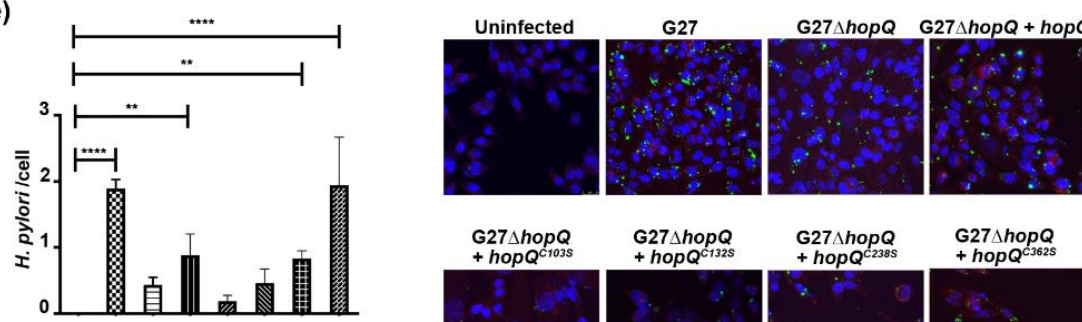

$\square$ Uninfected

m $\mathrm{G} 27$

口G27 $\triangle h O p Q$

m27 $\mathrm{GhopQ+hopQ^{ \textrm {c } 1 0 3 }}$ $\Delta \mathrm{G} 27 \Delta h o p Q+h o p Q^{c 132 s}$

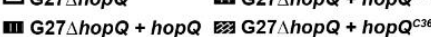
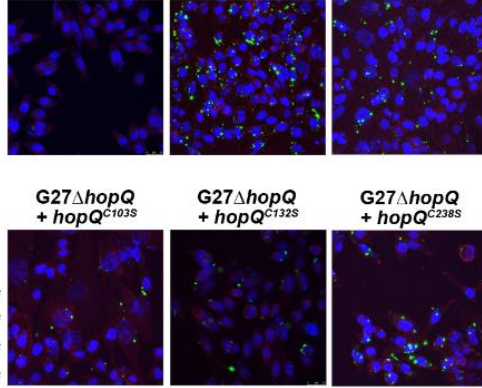

\section{.}

(f)

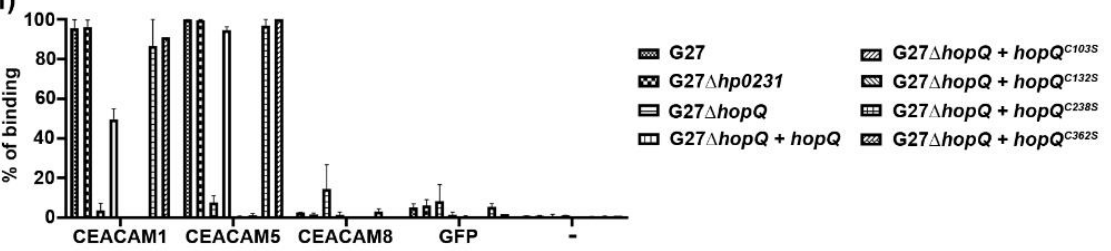

Figure 2. An intact CL1 disulfide in HopQ is crucial for CEACAM1 binding. (a) Western blot showing pull down pattern of recombinant CEACAM1 using the wild type H. pylori strain G27, G27 $\Delta$ hopQ, or G27 $\Delta$ hopQ strains that are complemented with either wild type or different mutant hop $Q$ alleles. (b) HopQ protein levels detected by Western blot in outer membrane extracts of different $H$. pylori strains. HpaA and gGT were used as positive and negative controls for the membrane preparation, respectively. (c) and (d) Flow cytometry cell-binding assay. Bar graph showing the percentage of $\mathrm{CHO}$ cells (carrying a vector control or expressing CEACAM1-4L) (c) or MKN28 cells (d) bound by fluorescently labelled H. pylori from panel (a). '-' denotes cells with no bacteria added. (e) Confocal microscopy and quantification of fluorescently labeled G27, G27 $\Delta h o p Q$, or G27 $\Delta$ hopQ strains complemented with either wild type or different mutant hopQ alleles binding to CHO-CEACAM1-4L (CC1) cells. Scale bar $25 \mu \mathrm{m}$. Magnification $=63 \times$. (f) Flow cytometry H. pylori binding assay. Bar graph showing the percentage of H. pylori binding to CEACAM-GFP. ${ }^{* *} p \leq 0.01,{ }^{* * *} p \leq 0.001,{ }^{* * * *} p \leq 0.0001$.

\subsection{H. pylori Oxidoreductase HP0231 is Not Required for HopQ Binding to Gastric Cells}

Knockout mutants of the H. pylori oxidoreductase HP0231 showed a CagA translocation defect, similar to what is seen in hopQ null mutants [3,7]. Since we found the CL1 disulfide to be important for HopQ functionality, we checked whether the CagA translocation defect seen in HP0231 mutants was a result of a compromised disulfide formation in HopQ. We first analyzed whether HP0231 influenced the expression of HopQ in different H. pylori strains, and observed that the deletion of $h p 0231$ did not affect the expression of HopQ (Figure 3a). To assess whether HopQ was still functional in the HP0231 KO strain, we performed pull-down assays using recombinant CEACAM1. We observed that HP0231-deficient bacteria were still able to pull down recombinant CEACAM1, whereas $\Delta$ hop $Q$ strains were unable to do so (Figure $3 b$ ). To substantiate these results, we performed binding assays to human gastric epithelial cells. The deletion of hop $Q$ led to a significant reduction in $H$. pylori binding to MKN28-CEACAM1 cells. In contrast, no changes in binding to the same cell lines were detected for strains lacking HP0231 (Figure 3c), confirming our previous observations [7]. We corroborated these results using confocal microscopy. We barely observed $H$. pylori binding to MKN28-CEACAM1 cells 
in the absence of HopQ (Figure 3d), while similar amounts of wild type and $\Delta h p 0231$ bacteria were observed to attach to the gastric epithelial cells.

Together, our results demonstrate that HP0231 does not influence H. pylori binding to gastric epithelial cells.

(a)

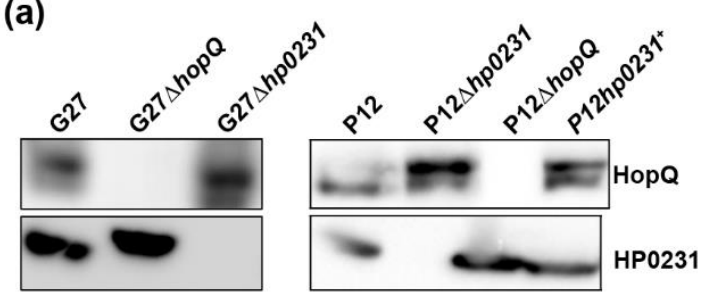

(c)

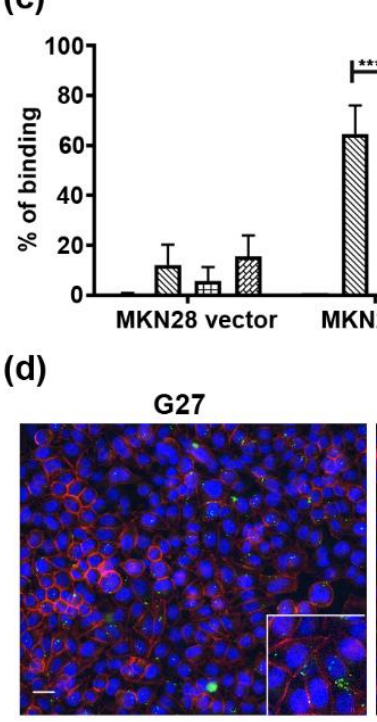

(b)

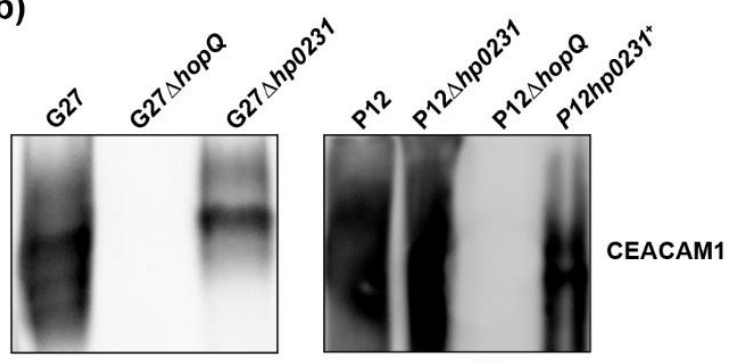

$\ddot{H}$

Figure 3. Deletion of HP0231 does not influence HopQ expression, stability or its CEACAM1 binding capacity. (a) Western blot of different H. pylori strains, probed with anti-HopQ and anti-HP0231 serum. (b) Pulldown of recombinant CEACAM1-Fc by several H. pylori strains. (c) Flow cytometry binding assay of same $H$. pylori strains to CEACAM1-deficient MKN28 cells, CEACAM1-4L-transfected MKN28 cells, CHO-CEACAM1-4L (CC1) cells, and AGS cells. '-' denotes cells with no bacteria added. (d) Immunofluorescence microscopy and quantification of G27 wild type, $\Delta$ hop $Q$ and $\Delta h p 0231 \mathrm{H}$. pylori strains binding to MKN28-CEACAM1-4L cells. Scale bar $25 \mu \mathrm{m}$. HPF, high-power field. ${ }^{*} p \leq 0.05$, ${ }^{* *} p \leq 0.01,{ }^{* * *} p \leq 0.001,{ }^{* * * *} p \leq 0.0001$.

\subsection{HopQ-CEACAM1 Mediated CagA Translocation is Dependent on the CL1 Disulfide}

We have previously shown that HopQ-CEACAM1 interaction is essential for CagA injection into host cells [3]. Once injected, CagA is phosphorylated and activates a number of signaling pathways that contribute to malignant transformation of the gastric mucosa. Having observed impaired binding of CL1 mutants to CEACAM1 (Figure 2), we assessed the ability of the different mutant strains to translocate CagA into gastric epithelial cells, by detecting phosphorylated CagA in cell lysates. We found that CL1 mutants were deficient in CagA translocation (Figure 4a,b), as was the $\Delta h p 0231$ mutant (Figure 4a,b), as previously published [7]. 
(a)

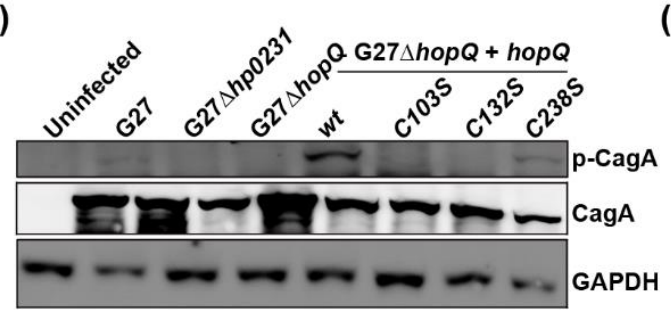

(b)

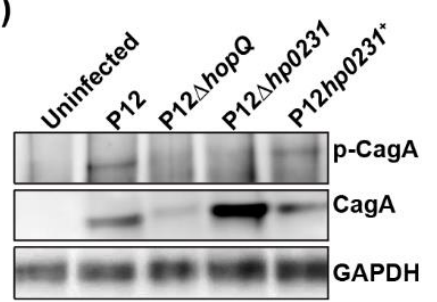

Figure 4. HopQ CL1 mutants are unable to translocate CagA. CagA, p-CagA, and GAPDH protein levels detected by Western blot in lysates of MKN28-CEACAM1-4L cells infected with different G27 (a) or P12 (b) H. pylori strains.

\section{Discussion}

Previously we described the importance of the HopQ-CEACAM interaction to $H$. pylori pathogenesis [3]. The co-complex structure between HopQ and CEACAM1 provided molecular details on the interaction and revealed the dependence on disulfide-clasped loops to form H-bonds and hydrophobic interactions with the N-terminal domain of CEACAM1 [5]. In this study, we demonstrate that the disulfide bond between residues $\mathrm{C} 103$ and $\mathrm{C} 132$, present in cysteine-clasped loop CL1, is required for CEACAM1 engagement by HopQ, whereas the disulfide bonds present in CL2 and 3are not. This is in contrast to what was reported in a recent paper [23], though consistent with our published co-complex structure [5]. As CL3 is located distant from the binding interface, no negative effect upon disruption of its disulfide is expected. However, the disulfide bond from CL2 is part of the HopQ-CEACAM1 binding interface and forms a platform that provides hydrophobic contacts to the interaction. It should be noted that CL2 does not contribute any direct H-bond to the interaction. This can explain why the loss of the CL2 disulfide bond does not affect HopQ-mediated binding, as any resulting conformational change would not directly disrupt $\mathrm{H}$-bonds, and CL2 can still perform its function as a supporting platform. Indeed, the only specific CL2 residues that interact with CEACAM1, Ile240 and Ile242, which form hydrophobic interactions with CEACAM1, are completely absent from the HopQ type II family, an alternative family of HopQ proteins that still maintains CEACAM binding [5]. In contrast, CL1 residue Tyr106 forms a key H-bond with CEACAM1 residue Thr56, and the loss of this H-bond has been shown to significantly disrupt the HopQ-CEACAM1 interaction [5]. Reducing the disulfide bond at the base of CL1 could alter its conformation to a non-functional state and abrogate this H-bond formation. CL1 also provides a scaffold for other structural elements of HopQ, namely the loops CL1-H3 and CL1-H4 (the loops between CL1 and $\alpha$-helices 3 and 4 , respectively). Disruption of these loops would result in a loss of up to 8 H-bonds, and can also help to explain the observed loss of binding. The finding that HopQ-CEACAM5 binding can be disrupted in a similar manner suggests that HopQ depends on similar strategies for binding both CEACAM1 and 5.

With the importance of the disulfide in CL1 to the HopQ-CEACAM interaction revealed, the next question was whether the Dsb-like oxidoreductase HP0231 is the enzyme responsible for the formation of this disulfide. We have previously shown that HP0231 is necessary for H. pylori virulence, as HP0231 mutants demonstrated reduced gastric colonization and were unable to translocate CagA into eukaryotic cells [7]. However, our results indicate that this is independent of HopQ, since the lack of HP0231 did not impair HopQ-CEACAM1 interaction, and thus the binding of H. pylori to epithelial cells. This finding is in contrast to a recent description of the interaction of HP0231 with HopQ, where HP0231 was thought to directly interact with and influence HopQ binding and subsequent CagA translocation [23]. The discrepancy in strains used or assay formats could explain the contrasting findings we observed, though in our assays we found that $\Delta h p 0231$ mutants in multiple strain backgrounds had no effect on HopQ-mediated binding in several assay settings. In the context of both studies, it could be proposed that HP0231 acts in concert with another Dsb-like protein. Two other Dsb-like proteins were reported in H. pylori-HP0595 (HpDsbI), which has been described as 
impacting HP0231 reoxidation [13], and HP0377, a DsbC-like protein which has been described as depending on HP0231 for redox homeostasis [14]. A possible link between HP0595 and HopQ function was ruled out, since HP0595-deficient bacteria were still able to translocate CagA into host cells [23]. HP0377, like HP0231, is localized in the periplasm, making this enzyme the most likely candidate for direct interaction with HopQ. However, the deletion of HP0377 is not possible, as it is an essential protein for H. pylori. Thus, it is difficult to assess its role in HopQ disulfide bond formation.

Preventing host-receptor interaction has been touted as a novel therapeutic approach to infectious disease. One approach that has proven successful in the past is the use of peptides to block pathogen binding. This has been demonstrated in approaches targeting viruses, such as Hepatitis B [24], human cytomegalovirus [25], influenza virus [26] and others [27], and, more recently, bacteria such as Mycobacterium tuberculosis [28]. Another approach is the use of antibodies to block the host-pathogen interaction [3], as well as nanobodies that overcome the limitations of traditional monoclonal antibodies and peptides as therapeutics $[29,30]$. An alternative approach that is prompted by identifying the importance of the CL1 disulfide bond in this study, and also avoids the limitations of other strategies, is to design small molecules that target and reduce the disulfide bond [31,32], thus preventing H. pylori binding; an approach that has previously been used to target HIV infection [33,34]. In this context, a previous study showed that binding of the H. pylori adhesin BabA to its cellular receptor Le ${ }^{\mathrm{b}}$ was impaired by the reduction in a conserved 8-residue cysteine-clasped loop in the adhesins glycan-binding site [8]. The treatment of $H$. pylori cells with the approved redox-active pharmaceutical $N$-acetyl cysteine (NAC) resulted in the loss of BabA-dependent binding to the gastric mucosa and the treatment of mice infected with $H$. pylori with a two-week regime of orally administered NAC resulted in a reduced bacterial colonization and gastric inflammation.

Studying the structural elements of HopQ-CEACAM interaction has allowed us to demonstrate a key and specific role for disulfide bonds in $H$. pylori virulence, but, more importantly, offered a broader view of the larger network of proteins that supports what initially seems a straightforward protein-protein interaction. Although HopQ has been proposed to interact with the T4SS [35], the impaired CagA-translocation observed in our study is a result of the binding defect induced by the mutations inserted. The clear importance of Dsb-like proteins to H. pylori infection, whether it be HopQ binding, CagA translocation or others, prompts further work to precisely identify their contributions and further our understanding of their roles.

Author Contributions: Conceptualization, M.G. and R.M.-L; methodology, Y.H., K.T., K.M., V.N., W.F., V.S., B.B.S., H.R.; formal analysis, Y.H., H.R., M.G. and R.M.-L.; writing-original draft preparation, Y.H. and R.M.-L.; writing-review and editing, Y.H. and R.M.-L.; supervision, R.M.-L. All authors have read and agreed to the published version of the manuscript.

Funding: This research was funded by DFG projects GE 2042/5-1 to M.G. and SI-1558/3-1 to B.B.S.

Acknowledgments: The authors thank Birgit Maranca-Hüwel and Bärbel Gobs-Hevelke (University of Duisburg-Essen, Germany) for excellent technical support. We also thank Christoph Hauck and Petra Zoll-Kiewitz (Universität Konstanz, Konstanz, Germany) for the CEACAM-GFP proteins.

Conflicts of Interest: The authors declare no conflict of interest. The funders had no role in the design of the study; in the collection, analyses, or interpretation of data; in the writing of the manuscript, or in the decision to publish the results.

\section{References}

1. Ishaq, S.; Nunn, L. Helicobacter pylori and gastric cancer: A state of the art review. Gastroenterol. Hepatol. Bed Bench 2015, 8, S6-S14.

2. Lindén, S.; Nordman, H.; Hedenbro, J.; Hurtig, M.; Borén, T.; Carlstedt, I. Strain- and blood group-dependent binding of Helicobacter pylori to human gastric MUC5AC glycoforms. Gastroenterology 2002, 123, 1923-1930. [CrossRef] [PubMed]

3. Javaheri, A.; Kruse, T.; Moonens, K.; Mejías-Luque, R.; Debraekeleer, A.; Asche, C.I.; Tegtmeyer, N.; Kalali, B.; Bach, N.C.; Sieber, S.A.; et al. Helicobacter pylori adhesin HopQ engages in a virulence-enhancing interaction with human CEACAMs. Nat. Microbiol. 2016, 2, 16189. [CrossRef] [PubMed] 
4. Königer, V.; Holsten, L.; Harrison, U.; Busch, B.; Loell, E.; Zhao, Q.; Bonsor, D.A.; Roth, A.; Kengmo-Tchoupa, A.; Smith, S.I.; et al. Erratum: Helicobacter pylori exploits human CEACAMs via HopQ for adherence and translocation of CagA. Nat. Microbiol. 2016, 2, 16188. [CrossRef] [PubMed]

5. Moonens, K.; Hamway, Y.; Neddermann, M.; Reschke, M.; Tegtmeyer, N.; Kruse, T.; Kammerer, R.; Mejías-Luque, R.; Singer, B.B.; Backert, S.; et al. Helicobacter pylori adhesin HopQ disrupts trans dimerization in human CEACAM s. EMBO J. 2018, 37, e98665. [CrossRef]

6. Bonsor, D.A.; Zhao, Q.; Schmidinger, B.; Weiss, E.; Wang, J.; Deredge, D.; Beadenkopf, R.; Dow, B.; Fischer, W.; Beckett, D.; et al. The Helicobacter pylori adhesin protein HopQ exploits the dimer interface of human CEACAMs to facilitate translocation of the oncoprotein CagA. EMBO J. 2018, 37, e98664. [CrossRef]

7. Zhong, Y.; Ander, F.; Kruse, T.; Schindele, F.; Jagusztyn-Krynicka, E.K.; Fischer, W.; Gerhard, M.; Mejías-Luque, R. Helicobacter pylori HP0231 influences bacterial virulence and is essential for gastric colonization. PLoS ONE 2016, 11, e0154643. [CrossRef]

8. Moonens, K.; Gideonsson, P.; Subedi, S.; Bugaytsova, J.; Romaõ, E.; Mendez, M.; Nordén, J.; Fallah, M.; Rakhimova, L.; Shevtsova, A.; et al. Structural Insights into Polymorphic ABO Glycan Binding by Helicobacter pylori. Cell Host Microbe 2016, 19, 55-66. [CrossRef]

9. Denoncin, K.; Collet, J.F. Disulfide bond formation in the bacterial periplasm: Major achievements and challenges ahead. Antioxid. Redox Signal. 2013, 19, 63-71. [CrossRef]

10. Shouldice, S.R.; Heras, B.; Walden, P.M.; Totsika, M.; Schembri, M.A.; Martin, J.L. Structure and function of DsbA, a key bacterial oxidative folding catalyst. Antioxid. Redox Signal. 2011, 14, 1729-1760. [CrossRef]

11. Berkmen, M. Production of disulfide-bonded proteins in Escherichia coli. Protein Expr. Purif. 2012, 82, $240-251$. [CrossRef] [PubMed]

12. Lester, J.; Kichler, S.; Oickle, B.; Fairweather, S.; Oberc, A.; Chahal, J.; Ratnayake, D.; Creuzenet, C. Characterization of Helicobacter pyloriHP0231 (DsbK): Role in disulfide bond formation, redox homeostasis and production of Helicobacter cystein-rich protein HcpE. Mol. Microbiol. 2015, 96, 110-133. [CrossRef] [PubMed]

13. Roszczenko, P.; Radomska, K.A.; Wywial, E.; Collet, J.F.; Jagusztyn-Krynicka, E.K. A Novel Insight into the Oxidoreductase Activity of Helicobacter pylori HP0231 Protein. PLoS ONE 2012, 7, e46563. [CrossRef]

14. Roszczenko, P.; Grzeszczuk, M.; Kobierecka, P.; Wywial, E.; Urbanowicz, P.; Wincek, P.; Nowak, E.; Jagusztyn-Krynicka, E.K. Helicobacter pylori HP0377, a member of the Dsb family, is an untypical multifunctional CcmG that cooperates with dimeric thioldisulfide oxidase HP0231. BMC Microbiol. 2015, 15, 135. [CrossRef]

15. Odenbreit, S.; Till, M.; Haas, R. Optimized BlaM-transposon shuttle mutagenesis of Helicobacter pylori allows the identification of novel genetic loci involved in bacterial virulence. Mol. Microbiol. 1996, 20, 361-373. [CrossRef]

16. Xiang, Z.; Censini, S.; Bayeli, P.F.; Telford, J.L.; Figura, N.; Rappuoli, R.; Covacci, A. Analysis of expression of CagA and VacA virulence factors in 43 strains of Helicobacter pylori reveals that clinical isolates can be divided into two major types and that CagA is not necessary for expression of the vacuolating cytotoxin. Infect. Immun. 1995, 63, 94-98. [CrossRef]

17. Romano, M.; Razandi, M.; Sekhon, S.; Krause, W.J.; Ivey, K.J. Human cell line for study of damage to gastric epithelial cells in vitro. J. Lab. Clin. Med. 1988, 111, 430-440.

18. Müller, M.M.; Klaile, E.; Vorontsova, O.; Singer, B.B.; Öbrink, B. Homophilic adhesion and CEACAM1-S regulate dimerization of CEACAM1-L and recruitment of SHP-2 and c-Src. J. Cell Biol. 2009, 187, 569-581. [CrossRef]

19. Tchoupa, A.K.; Lichtenegger, S.; Reidl, J.; Hauck, C.R. Outer membrane protein P1 is the CEACAM-binding adhesin of Haemophilus influenzae. Mol. Microbiol. 2015, 98, 440-455. [CrossRef]

20. Kuespert, K.; Weibel, S.; Hauck, C.R. Profiling of bacterial adhesin-host receptor recognition by soluble immunoglobulin superfamily domains. J. Microbiol. Methods 2007, 68, 478-485. [CrossRef]

21. Kuespert, K.; Hauck, C.R. Characterizing host receptor recognition by individual bacterial pathogens. Methods Mol. Biol. 2009, 470, 57-65. [PubMed]

22. Muturi, H.T.; Dreesen, J.D.; Nilewski, E.; Jastrow, H.; Giebel, B.; Ergun, S.; Singer, B.B. Tumor and Endothelial Cell-Derived Microvesicles Carry Distinct CEACAMs and Influence T-Cell Behavior. PLoS ONE 2013, 8, e74654. [CrossRef] [PubMed] 
23. Grzeszczuk, M.J.; Bocian-Ostrzycka, K.M.; Banaś, A.M.; Roszczenko-Jasinska, P.; Malinowska, A.; Stralova, H.; Haas, R.; Meyer, T.F.; Jagusztyn-Krynicka, E.K. Thioloxidoreductase HP0231 of Helicobacter pylori impacts HopQ-dependent CagA translocation. Int. J. Med. Microbiol. 2018, 308, 977-985. [CrossRef] [PubMed]

24. Ye, X.; Zhou, M.; He, Y.; Wan, Y.; Bai, W.; Tao, S.; Ren, Y.; Zhang, X.; Xu, J.; Liu, J.; et al. Efficient Inhibition of Hepatitis B Virus Infection by a preS1-binding Peptide. Sci. Rep. 2016, 6, 29391. [CrossRef]

25. Melnik, L.I.; Garry, R.F.; Morris, C.A. Peptide inhibition of human cytomegalovirus infection. Virol. J. 2011, 8, 76. [CrossRef]

26. Rajik, M.; Omar, A.R.; Ideris, A.; Hassan, S.S.; Yusoff, K. A novel peptide inhibits the influenza virus replication by preventing the viral attachment to the host cells. Int. J. Biol. Sci. 2009, 5, 543-548. [CrossRef]

27. Krepstakies, M.; Lucifora, J.; Nagel, C.-H.; Zeisel, M.B.; Holstermann, B.; Hohenberg, H.; Kowalski, I.; Gutsmann, T.; Baumert, T.F.; Brandenburg, K.; et al. A New Class of Synthetic Peptide Inhibitors Blocks Attachment and Entry of Human Pathogenic Viruses. J. Infect. Dis. 2012, 205, 1654-1664. [CrossRef]

28. Sánchez-Barinas, C.D.; Ocampo, M.; Tabares, L.; Bermúdez, M.; Patarroyo, M.A.; Patarroyo, M.E. Specific Binding Peptides from Rv3632: A Strategy for Blocking Mycobacterium tuberculosis Entry to Target Cells? Biomed Res. Int. 2019, 2019, 8680935. [CrossRef]

29. Aldeghaither, D.; Smaglo, B.G.; Weiner, L.M. Beyond peptides and mAbs-Current status and future perspectives for biotherapeutics with novel constructs. J. Clin. Pharmacol. 2015, 55, S4-S20. [CrossRef]

30. Siontorou, C.G. Nanobodies as novel agents for disease diagnosis and therapy. Int. J. Nanomed. 2013, 8, 4215-4227. [CrossRef]

31. Chiu, J.; Hogg, P.J. Allosteric disulfides: Sophisticated molecular structures enabling flexible protein regulation. J. Biol. Chem. 2019, 294, 2949-2960. [CrossRef] [PubMed]

32. Hallenbeck, K.K.; Turner, D.M.; Renslo, A.R.; Arkin, M.R. Targeting Non-Catalytic Cysteine Residues Through Structure-Guided Drug Discovery. Curr. Top. Med. Chem. 2017, 17, 4-15. [CrossRef] [PubMed]

33. Martin, G.; Burke, B.; Thaï, R.; Dey, A.K.; Combes, O.; Heyd, B.; Geonnotti, A.R.; Montefiori, D.C.; Kan, E.; Lian, Y.; et al. Stabilization of HIV-1 envelope in the CD4-bound conformation through specific cross-linking of a CD4 mimetic. J. Biol. Chem. 2011, 286, 21706-21716. [CrossRef]

34. Cerutti, N.; Mendelow, B.V.; Napier, G.B.; Papathanasopoulos, M.A.; Killick, M.; Khati, M.; Stevens, W.; Capovilla, A. Stabilization of HIV-1 gp120-CD4 receptor complex through targeted interchain disulfide exchange. J. Biol. Chem. 2010, 285, 25743-25752. [CrossRef] [PubMed]

35. Tegtmeyer, N.; Harrer, A.; Schmitt, V.; Singer, B.B.; Backert, S. Expression of CEACAM1 or CEACAM5 in AZ-521 cells restores the type IV secretion deficiency for translocation of CagA by Helicobacter pylori. Cell. Microbiol. 2019, 21, e12965. [CrossRef] [PubMed]

(C) 2020 by the authors. Licensee MDPI, Basel, Switzerland. This article is an open access article distributed under the terms and conditions of the Creative Commons Attribution (CC BY) license (http://creativecommons.org/licenses/by/4.0/). 